\title{
Mortalidad en México. Algunas consideraciones sobre los diferenciales urbano-rurales*
}

\section{Sergio Camposortega Cruz**}

En este trabajo se analizan algunos aspectos relacionados con los diferenciales de mortalidad urbano-rurales en México, tales como la disponibilidad, las ventajas y limitaciones de las diversas fuentes de información y la disparidad de niveles y tendencias según algunos indicadores de confiabilidad aceptable, en particular los que se derivan de las recientes encuestas demográficas del país. Los resultados confirman la relación inversa entre tamaño de localidad y mortalidad y muestran el ensanchamiento de los diferenciales con el paso del tiempo.

\section{Introducción}

El objetivo del presente trabajo es analizar algunas características de la mortalidad en México relacionadas con los diferenciales urbano-rurales. Para ello se analizan, en primer lugar, las fuentes de información en cuanto a disponibilidad, calidad de la información y utilidad para el estudio de la mortalidad por tamaño de la localidad; posteriormente se presenta, como marco de referencia, la evolución de la mortalidad en México y por último se exami. nan, con base en los indicadores que consideramos más confiables, algunas diferencias de la mortalidad en relación con el tamaño de la localidad.

\section{Fuentes de información}

México presenta, en comparación con los países subdesarrollados, una situación privilegiada en lo que se refiere a las fuentes de información demográfica. En efecto, los censos generales de población que se remontan a fines del siglo XIX y que a partir de 1900 han sido levantados cada diez años, proporcionan cada vez

* Versión corregida (septiembre de 1989) de la ponencia presentada en e] Seminario "Demografía y Urbanización en México", organizado por el Centro de Es. tudios Demográficos y de Desarrollo Urbano de El Colegio de México en diciembre de 1987.

** Profesor-investigador del Centro de Estudios Demográficos y de Desarrollo Urbano de El Colegio de México. 
con mayor certeza las características demográficas de la población; por su parte, el Registro Civil, fundado a mediados del siglo XIX, desde los años veinte publica ininterrumpidamente series estadísticas nacionales y estatales sobre nacimientos y defunciones por sexo y edad. Por último, el levantamiento en años recientes de varias encuestas de tipo demográfico ${ }^{1}$ ha enriquecido considerablemente el acervo de datos disponibles para el estudio de la mortalidad en México.

Sin embargo, los datos nacionales en que se basan los parámetros de mortalidad [estructuras por edad y sexo, nacimientos y defunciones por edad y sexo] registran diversos tipos de error, que se ven acrecentados en los niveles regional, estatal, por tamaño de localidad o según la distinción de otras variables. En términos generales, se ha establecido que:

- los datos censales sobre la estructura por edad y sexo de la población adolecen de problemas de subregistro, particularmente en los menores de 5 años y en los hombres entre 15 y 35 años, ${ }^{2}$ y de mala declaración de la edad, en especial alta preferencia por los dígitos 0 y 5 y exageración de la edad en las personas ancianas ${ }^{3}$ (Camposortega, 1984; Monterrubio, y Camposortega, 1986; Camposortega, 1988a y 1988b);

- las estadísticas vitales sobre nacimientos, aunque tradicionalmente completas (Lailson, et al., 1979), presentan el problema del registro tardío, que a raíz del descenso de la fecundidad se ha traducido en un sobrerregistro de nacimientos; ${ }^{4}$

1 Entre las más importantes conviene destacar: la Encuesta Mexicana de Fecundidad levantada en 1976 por la Dirección General de Estadística, dentro del programa denominado Encuesta Mundial de Fecundidad; la Encuesta Nacional de Prevalencia en el Uso de Métodos Anticonceptivos con Módulo Fecundidad/Mortalidad de 1979, realizada por la Coordinación General del Programa Nacional de Planificación Familiar y la Encuesta Nacional Sociodemográfica, levantada en 1982 por el Consejo Nacional de Población, y la Encuesta Nacional de Fecundidad y Salud de 1987, llevada a cabo por la Dirección General de Planificación Familiar de la Secretarfa de Salud.

${ }^{2}$ Las diferencias entre las cifras censales y las estimadas a partir de estadísticas vitales en el grupo de 0 a 4 años son de] $14.1 \%$ en $1940,13.6 \%$ en 1950 , $13.5 \%$ en $1960,11.0 \%$ en 1970 y $17.4 \%$ en 1980 , lo que da una idea del subregistro censal.

${ }^{3}$ Las diferencias entre los datos censales y las estimaciones provenientes de los censos anteriores y las probabilidades de muerte en la población mayor de 75 años son del $30 \%$ en 1950, 25\% en 1960 y $18 \%$ en 1970 (Camposortega, 1988b).

${ }^{4}$ De tal suerte, hacia 1980 la tasa de fecundidad global nacional se estimaba 
- las estadísticas sobre defunciones sufren omisiones, en especial en la mortalidad infantil (Cordero, 1968; Aguirre y Camposortega, 1981; Camposortega, 1988a) y en las muertes rurales (Arriaga, 1967) y deficiente declaración de la edad (Camposortega, 1988a y 1988b);

- en general, la calidad de los datos ha mejorado paulatinamente (Camposortega, 1988a).

Estas consideraciones ilustran tanto las restricciones a que están sujetas las estimaciones de mortalidad, como el cuidado que debe tenerse al utilizar las cifras, si no quiere llegarse a conclusiones equivocadas.

Los parámetros de mortalidad que se analizan en el siguiente apartado para el caso mexicano parten de una detallada evaluación de los datos censales y de estadísticas vitales y hacen uso de las recientes encuestas demográficas (Camposortega, 1989 y 1988a). Estas cifras, en términos generales, apenas difieren de las estimaciones elaboradas previamente por otros autores, si bien las esperanzas de vida resultantes son poco menores que las que se calculan con los datos brutos [cuadro 1].

En el caso particular de las estadísticas urbano-rurales, la situación resulta más complicada. En primer lugar, porque se cuenta con mucho menos información; sólo a partir de 1975 exister. datos publicados sobre defunciones por edad, sexo y tamaño de localidad (Núñez y Moreno, 1986); en segundo, porque la calidad de la información relacionada con el tamaño de localidad es todavía más deficiente. Al respecto, se ha señalado incluso que las deficiencias en los datos estadísticos imposibilitan el cálculo de las diferencias urbano-rurales (CEED, 1981; Benítez, 1962).

Analizaremos a continuación los principales datos que permiten estimar la mortalidad urbana y rural.

Sobre las estadísticas vitales se puede establecer que, en algunos casos, los datos sobre mortalidad general -cálculo de tasas brutas- sufren altos grados de omisión, como en Baja California en 1959-1961, cuya tasa rural era del orden de 4.2 defunciones por cada mil habitantes, contra $9.2 \%$ en el área urbana, o en Tamaulipas en el mismo año, con una tasa rural de 5.1\% y una urbana de $11.2 \%$ (Arriaga, 1967), como se aprecia en el cuadro 2.

Es probable que también exista el problema de sobreestimación de la mortalidad urbana por la migración de población enfer-

en 5.0 hijos, según las estadísticas vitales, en tanto que según la Encuesta Nacional Demográfica en sólo 4.4 hijos (Ordorica y Medina, 1986). 
CUADRO 1

México: algunos parámetros de las tablas de mortalidad corregidas, 1940-1980

\begin{tabular}{|c|c|c|c|c|c|c|c|c|}
\hline Periodo & $e[0]$ & $e[1]$ & $e(15)$ & $e[30)$ & $e(65)$ & $\begin{array}{c}q(0,1) \\
0 \% 00\end{array}$ & $\begin{array}{c}q(1,4) \\
0 \% 00\end{array}$ & $\begin{array}{c}q(25,20) \\
0 \% 00\end{array}$ \\
\hline \multicolumn{9}{|c|}{ Los dos sexos } \\
\hline $1939-1941$ & 40.35 & 47.14 & 42.91 & 32.48 & 10.89 & 162.95 & 131.64 & 218.28 \\
\hline $1949-1951$ & 48.66 & 54.83 & 48.09 & 36.34 & 12.06 & 129.29 & 91.01 & 151.61 \\
\hline $1959-1961$ & 57.81 & 62.52 & 52.46 & 39.70 & 13.20 & 90.25 & 44.25 & 103.69 \\
\hline $1969-1971$ & 60.98 & 65.03 & 54.17 & 41.03 & 13.78 & 76.84 & 34.41 & 74.87 \\
\hline 1979-1981 & 66.18 & 68.88 & 56.31 & 42.98 & 14.88 & 53.10 & 13.69 & 74.87 \\
\hline \multicolumn{9}{|l|}{ Hombres } \\
\hline $1939-1941$ & 39.12 & 46.57 & 41.66 & 31.45 & 10.68 & 178.88 & 123.76 & 235.10 \\
\hline 1949-1951 & 46.74 & 53.59 & 46.51 & 34.98 & 11.63 & 144.61 & 88.07 & 167.48 \\
\hline $1959-1961$ & 55.99 & 61.22 & 51.02 & 38.53 & 12.91 & 100.55 & 43.80 & 117.27 \\
\hline $1969-1971$ & 59.01 & 63.49 & 52.45 & 39.68 & 13.32 & 85.41 & 32.55 & 102.66 \\
\hline 1979-1981 & 63.16 & 66.06 & 53.48 & 40.76 & 14.07 & 58.41 & 13.12 & 99.53 \\
\hline \multicolumn{9}{|l|}{ мuјетеs } \\
\hline $1939-1941$ & 41.65 & 47.72 & 44.21 & 33.52 & 11.09 & 146.23 & 139.59 & 201.13 \\
\hline 1949-1951 & 50.68 & 56.10 & 49.71 & 37.70 & 12.46 & 113.20 & 93.98 & 135.62 \\
\hline $1959-1961$ & 59.73 & 63.86 & 53.94 & 40.90 & 13.48 & 79.43 & 44.71 & 89.82 \\
\hline $1969-1971$ & 63.06 & 66.63 & 55.95 & 42.42 & 14.22 & 67.84 & 36.32 & 72.94 \\
\hline $1979-1981$ & 69.39 & 71.85 & 59.29 & 45.26 & 15.66 & 47.52 & 14.28 & 49.45 \\
\hline
\end{tabular}

ma, que en caso de fallecimiento quizá es registrada en las zonas de atención (Partida, 1982).

Los recientes datos por edad y sexo han permitido el cálculo de tasas específicas (cuadro 3); sin embargo, los resultados no parecen muy confiables: la estructura es un tanto irregular (gráfica 1) y los niveles de mortalidad derivados de cada tasa, según el patrón latinoamericano, no concuerdan con las estimaciones nacionales ni parecen adaptarse a dicho patrón que, como ya se ha probado, refleja el comportamiento nacional (Camposortega, 1988a). La gráfica 2 estaría mostrando la existencia de un mayor subregistro conforme avanza la edad.

Otra posibilidad de información la constituye el acervo de encuestas demográficas, en particular:

- La Encuesta Mexicana de Fecundidad de 1976 (SPP, 1979), que permite estimar la mortalidad en las primeras edades en las áreas urbana y rural, así como en cada una de las tres 
CUADRO 2

México: tasas brutas de mortalidad por entidad federativa y lugar de residencia, 1949-1951, 1959-1961, 1969-1971 y 1979-1980

\begin{tabular}{|c|c|c|c|c|c|c|c|c|c|c|c|c|}
\hline \multirow[b]{2}{*}{ Entidad federativa } & \multicolumn{3}{|c|}{$1949-1951$} & \multicolumn{3}{|c|}{$1959-1961$} & \multicolumn{3}{|c|}{19691971} & \multicolumn{3}{|c|}{$1979-1980$} \\
\hline & Total & Urbano & Rural & Total & Urbano & Rural & Total & Urbano & Rural & Total & Urbano & Rural \\
\hline México & 17.1 & 16.7 & 17.4 & 11.5 & 11.1 & 11.9 & 9.7 & 9.6 & 9.9 & 6.4 & 6.1 & 7.1 \\
\hline Aguascalientes & 19.0 & 20.2 & 17.6 & 12.3 & 12.3 & 12.2 & 10.2 & 10.9 & 9.1 & 6.5 & 6.2 & 7.2 \\
\hline $\begin{array}{l}\text { Baja California } \\
\text { Baja California }\end{array}$ & 14.4 & 18.3 & 9.9 & 8.0 & 9.3 & 4.2 & 7.7 & 8.1 & 5.6 & 5.7 & 5.4 & 7.7 \\
\hline Sur & 11.8 & 19.0 & 8.1 & 8.1 & 13.0 & 5.3 & 7.1 & 7.5 & 6.6 & 5.6 & 6.2 & 4.2 \\
\hline Campeche & 12.6 & 13.7 & 11.4 & 9.8 & 10.5 & 8.8 & 8.0 & 9.2 & 5.9 & 5.1 & 5.6 & 4.0 \\
\hline Coahuila & 15.7 & 17.5 & 13.5 & 11.1 & 11.8 & 9.6 & 9.9 & 10.8 & 7.6 & 6.2 & 6.4 & 5.2 \\
\hline Colima & 17.2 & 19.5 & 15.3 & $12: 6$ & 14.6 & 9.5 & 9.9 & 10.6 & 8.4 & 7.1 & 7.7 & 5.5 \\
\hline Chiapas & 15.9 & 18.4 & 15.4 & 12.2 & 13.2 & 11.9 & 11.4 & 13.1 & 10.7 & 5.9 & 7.4 & 5.1 \\
\hline Chihuahua & 14.3 & 18.2 & 11.6 & 10.0 & 10.8 & 8.8 & 8.5 & 8.9 & 7.7 & 6.5 & 6.8 & 6.0 \\
\hline Distrito Federal & 17.3 & 16.7 & 26.7 & 10.0 & 10.0 & 11.7 & 9.4 & 9.5 & 5.6 & 5.4 & 5.4 & 0.0 \\
\hline Durango & 13.1 & 18.9 & 11.1 & 9.4 & 13.0 & 7.4 & 7.4 & 10.2 & 5.4 & 5.4 & 6.5 & 4.3 \\
\hline Guanajuato & 21.6 & 21.5 & 21.8 & 13.5 & 14.2 & 12.8 & 11.2 & 12.4 & 9.9 & 7.7 & 7.8 & 7.4 \\
\hline Guerrero & 14.1 & 16.9 & 13.6 & 10.0 & 12.6 & 9.2 & 8.1 & 8.9 & 7.6 & 6.2 & 6.2 & 6.2 \\
\hline Hidalgo & 20.3 & 19.1 & 20.7 & 14.7 & 12.5 & 15.3 & 11.6 & 9.9 & 12.3 & 8.3 & 9.4 & 7.8 \\
\hline Jalisco & 17.3 & 19.8 & 15.3 & 10.9 & 11.4 & 10.3 & 9.6 & 10.2 & 8.4 & 6.6 & 6.7 & 6.5 \\
\hline México & 22.9 & 18.0 & 24.6 & 15.1 & 9.4 & 18.5 & 10.1 & 6.2 & 16.4 & 6.0 & 4.3 & 12.5 \\
\hline Michoacán & 15.3 & 19.6 & 13.5 & 9.6 & 11.0 & 8.7 & 8.3 & 10.8 & 6.2 & 7.1 & 8.4 & 5.7 \\
\hline Morelos & 17.1 & 19.9 & 16.1 & 10.3 & 10.5 & 10.0 & 8.4 & 8.2 & 8.8 & 6.0 & 6.0 & 5.9 \\
\hline Nayarit & 14.3 & 20.8 & 11.2 & 10.8 & 13.8 & 8.6 & 8.1 & 9.7 & 6.5 & 5.1 & 5.7 & 4.2 \\
\hline Nuevo León & 12.8 & 15.4 & 10.2 & 8.3 & 8.5 & 7.7 & 7.1 & 7.2 & 6.6 & 4.8 & 4.1 & 9.7 \\
\hline Oaxaca & 21.6 & 19.9 & 22.2 & 15.8 & 13.3 & 16.6 & 14.4 & 13.1 & 14.9 & 10.2 & 9.2 & 10.6 \\
\hline Puebla & 22.8 & 18.7 & 24.8 & 16.1 & 12.0 & 18.8 & 13.7 & 11.2 & 15.9 & 9.5 & 8.0 & 11.6 \\
\hline Querétaro & 20.2 & 20.3 & 20.6 & 14.0 & 11.9 & 14.9 & 11.3 & 9.3 & 12.4 & 7.2 & 6.1 & 8.1 \\
\hline
\end{tabular}


CUADRO 2 (continuación)

\begin{tabular}{|c|c|c|c|c|c|c|c|c|c|c|c|c|}
\hline \multirow[b]{2}{*}{ Entidad federativa } & \multicolumn{3}{|c|}{$1949-1951$} & \multicolumn{3}{|c|}{$1959-1961$} & \multicolumn{3}{|c|}{$1969-1971$} & \multicolumn{3}{|c|}{$1979-1980$} \\
\hline & Total & Urbano & Rural & Total & Urbano & Rural & Total & Urbano & Rural & Total & Urbano & Rural \\
\hline Quintana Roo & 10.1 & 15.6 & 8.2 & 5.0 & 5.4 & 4.8 & 5.9 & 7.8 & 4.7 & 4.4 & 4.4 & 4.3 \\
\hline San Luis Potosí & 17.1 & 19.9 & 16.2 & 12.6 & 12.7 & 12.6 & 10.9 & 12.0 & 10.2 & 6.0 & 5.7 & 6.4 \\
\hline Sinaloa & 11.3 & 17.0 & 9.4 & 8.4 & 10.3 & 7.2 & 6.9 & 7.2 & 6.6 & 5.0 & 5.0 & 4.9 \\
\hline Sonora & 13.6 & 16.9 & 11.8 & 10.0 & 10.8 & 8.9 & 8.5 & 9.0 & 7.4 & 6.3 & 6.5 & 5.8 \\
\hline Tabasco & 12.9 & 14.1 & 12.7 & 10.4 & 11.1 & 10.2 & 8.6 & 10.3 & 7.8 & 6.7 & 7.2 & 6.4 \\
\hline Tamaulipas & 12.2 & 16.7 & 7.9 & 8.7 & 11.2 & 5.1 & 7.1 & 8.3 & 4.4 & 5.7 & 6.2 & 4.4 \\
\hline Tlaxcala & 23.9 & 19.6 & 26.5 & 15.6 & 12.1 & 18.3 & 13.6 & 12.7 & 14.5 & 6.3 & 5.5 & 7.5 \\
\hline Veracruz & 13.9 & 17.7 & 12.1 & 10.3 & 11.7 & 9.3 & 9.0 & 10.2 & 7.8 & 6.0 & 7.0 & 5.1 \\
\hline Yucatán & 16.3 & 15.9 & 16.9 & 12.5 & 12.0 & 13.4 & 10.2 & 10.4 & 9.8 & 7.5 & 7.6 & 7.4 \\
\hline Zacatecas & 18.1 & 19.7 & 17.6 & 11.1 & 12.7 & 10.4 & 9.2 & 11.8 & 8.0 & 6.0 & 7.4 & 5.1 \\
\hline
\end{tabular}

Fuentes: 1949-1951 y 1959-1961, Arriaga E. (1967). 1969-1971 y 1979-1981, cálculos propios con base en datos de la Dirección General de Estadístice. 
CUADRO 3

México: tasas específicas de mortalidad masculina adulta por condición de residencia según edad, 1975-1979 (\%a)

\begin{tabular}{|c|c|c|c|c|c|c|c|c|c|c|}
\hline \multirow[b]{2}{*}{ Edad } & \multicolumn{2}{|c|}{1975} & \multicolumn{2}{|c|}{1976} & \multicolumn{2}{|c|}{1977} & \multicolumn{2}{|c|}{1978} & \multicolumn{2}{|c|}{1979} \\
\hline & Rural & $\overline{\text { Urbana }}$ & Rural & Urbana & Rural & Urbana & Rural & Urbana & Rural & Urbana \\
\hline $15-19$ & 1.9 & 1.8 & 1.8 & 1.7 & 2.1 & 1.7 & 1.9 & 1.7 & 2.4 & 1.6 \\
\hline $20-24$ & 3.7 & 3.5 & 3.5 & 2.8 & 3.9 & 2.9 & 3.8 & 2.9 & 4.4 & 2.8 \\
\hline $25-29$ & 5.2 & 3.9 & 4.9 & 3.6 & 4.5 & 3.7 & 4.8 & 3.6 & 5.2 & 3.7 \\
\hline $30-34$ & 5.3 & 4.1 & 5.0 & 4.1 & 5.2 & 4.2 & 4.6 & 3.9 & 5.1 & 3.9 \\
\hline $35-39$ & 7.3 & 5.8 & 6.7 & 5.7 & 6.5 & 5.8 & 5.7 & 5.4 & 6.4 & 5.2 \\
\hline $40-44$ & 6.6 & 6.4 & 6.0 & 6.4 & 6.4 & 6.1 & 6.3 & 5.8 & 7.0 & 6.0 \\
\hline $45-49$ & 8.7 & 8.8 & 8.2 & 8.4 & 7.5 & 8.1 & 8.2 & 7.7 & 8.7 & 7.6 \\
\hline $50-54$ & 10.5 & 12.8 & 9.9 & 11.6 & 9.9 & 10.8 & 9.5 & 10.1 & 9.8 & 10.2 \\
\hline $55-59$ & 13.6 & 16.3 & 13.5 & 16.3 & 12.9 & 15.6 & 13.0 & 15.0 & 14.1 & 15.8 \\
\hline $60-64$ & 19.6 & 23.5 & 17.2 & 22.2 & 16.3 & 20.0 & 18.3 & 19.1 & 17.8 & 19.2 \\
\hline $65-69$ & 24.2 & 32.6 & 24.2 & 35.4 & 21.1 & 32.6 & 26.4 & 30.4 & 28.2 & 32.0 \\
\hline $70-74$ & 36.1 & 53.1 & 33.7 & 49.2 & 27.7 & 44.2 & 33.4 & 43.2 & 33.8 & 41.3 \\
\hline $75-79$ & 53.2 & 74.9 & 56.5 & 81.4 & 39.8 & 75.4 & 47.3 & 72.9 & 49.8 & 76.7 \\
\hline 80 y. más & 134.4 & 163.0 & 135.6 & 163.1 & 106.9 & 141.2 & 115.1 & 134.8 & 121.5 & 133.2 \\
\hline
\end{tabular}

Fuente: Núñez y Morano (1986), p. 42. 
Gráfica 1

México: tasas de mortalidad masculina

(Promedio 1975-1979)

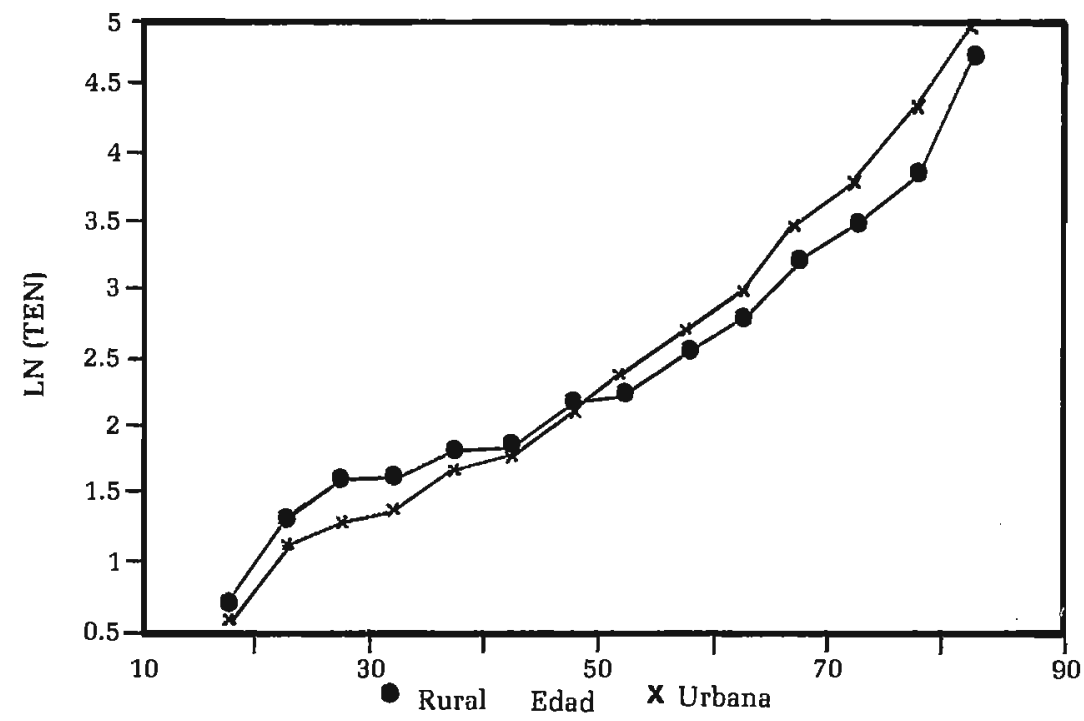

zonas metropolitanas del país (México, Guadalajara y Monterrey) a partir del método directo y de los métodos sobre hijos nacidos vivos e hijos sobrevivientes (Brass, 1971; Camposortega, 1989):

- La Encuesta Nacional de Prevalencia en el Uso de Métodos Anticonceptivos de 1979 (CPNPF, 1980a y 1980b), que proporciona la información necesaria para la aplicación de los métodos directos de estimación de la mortalidad en las primeras edades (hijos nacidos vivos e hijos sobrevivientes) y de la mortalidad adulta (orfandad materna y paterna) (Brass, y Hill, 1973), para las zonas urbana y rural.

- La Encuesta Rural de Planificación Familiar de 1981 (JSPF, 1983a y 1983b), que permite estimar la mortalidad en las primeras edades y en las edades adultas [métodos de orfandad y viudez) en las localidades menores de 2500 habitantes [Hill, 1977).

- La Encuesta Nacional de Fecundidad y Salud de 1987 [DGPF, 1988], que proporciona los datos necesarios para la 
Gráfica 2

México: e[o] correspondiente, hombres

(Patrón latinoamericano 1975-1979)

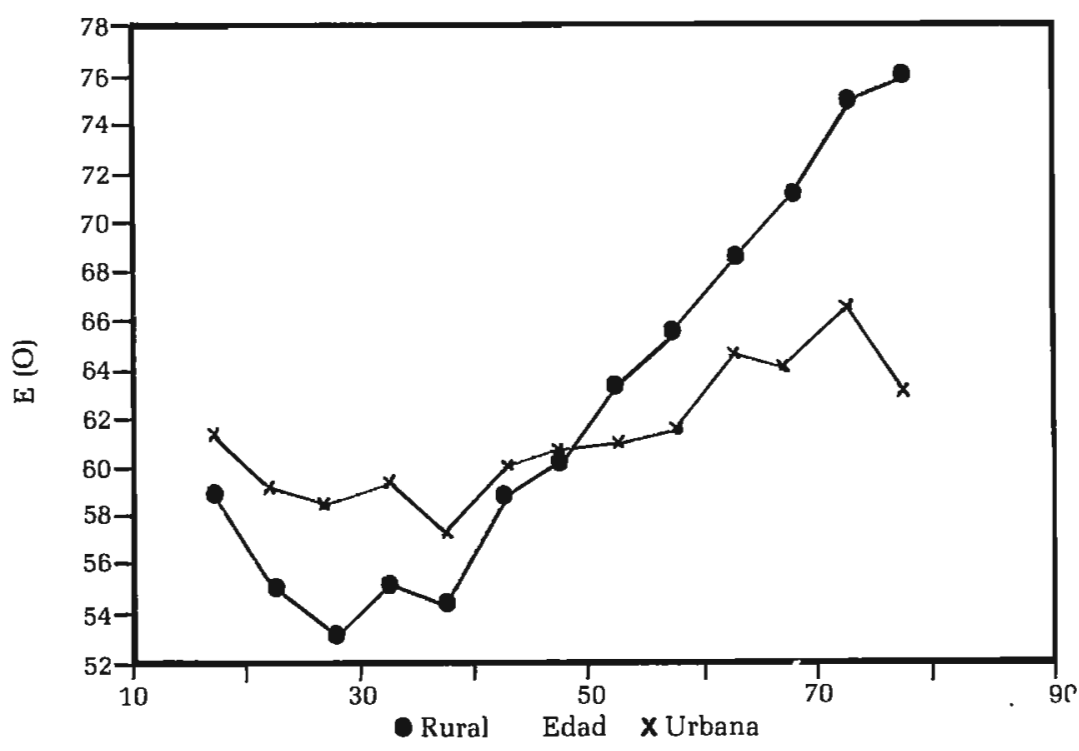

estimación directa e indirecta de la mortalidad en las primeras edades.

En términos generales se ha establecido que la calidad de la información proveniente de estas encuestas es aceptable, en particular, la de la Encuesta Mexicana de Fecundidad (Ordorica y Potter, 1980). Existen, sin embargo, ciertos factores que restan confiabilidad a las estimaciones por tamaño de docalidad:

-El primero se refiere al tamaño de la muestra de las encuestas ya que, salvo en la de 1981, la clasificación urbano-rural resulta de una subdivisión de la muestra, lo que obstaculiza los cálculos, puesto que al ser la defunción un suceso raro requiere de un número considerable de entrevistas. Los diagramas de Lexis referentes a las ciudades de Guadalajara y Monterrey sobre defunciones en las primeras edades, captadas en la Encuesta Mexicana de Fecundidad, son clara muestra de las dificultades de estimación (gráfica 3).

- Otro factor es el relativo a la robustez de los procedimien- 
Gréfica 3

Guadalajara: Defunciones, 1971-1978
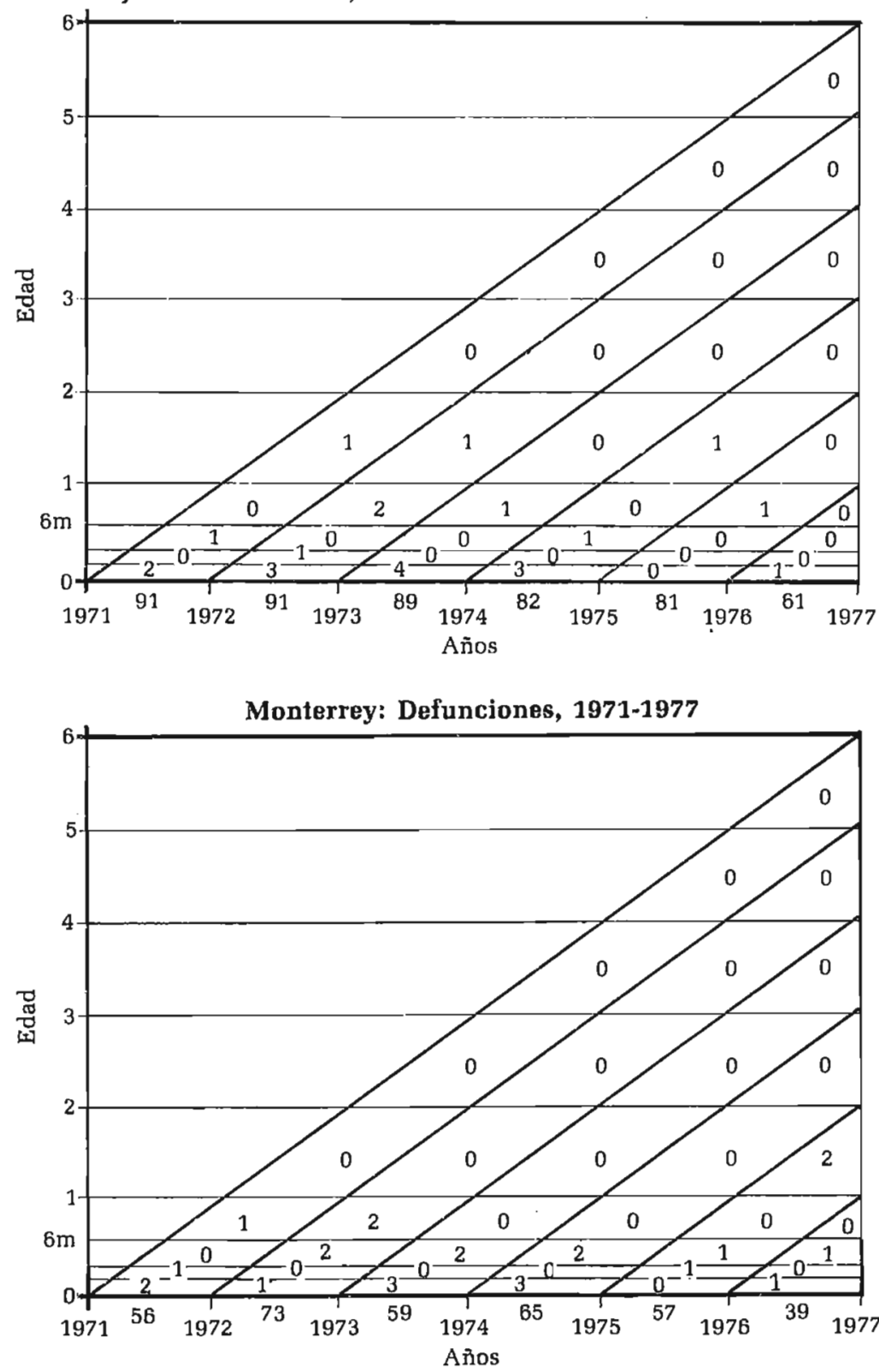
tos de estimación de la mortalidad adulta, los cuales son poco flexibles y en las aplicaciones prácticas no han proporcionado resultados confiables.

Finalmente, el censo de población de 1980 es otra posibilidad para la estimación de la mortalidad en las primeras edades según tamaño de localidad (INEGI, 1987) e incluso para cada uno de los municipios del país. Sin embargo, las deficiencias de estos datos impiden estimar la mortalidad en forma razonable. En escala nacional ésta se sobrestima entre un 14 y 23 por ciento [Camposortega, 1986); en los ámbitos estatal y municipal es común encontrar, además de la sobreestimación, resultados verdaderamente absurdos, como en los casos de Durango, Chihuahua, Sinaloa y Veracruz. ${ }^{5}$

Una de las causas de estas deficiencias se encuentra en el tratamiento de los no especificados en la información sobre hijos nacidos vivos, ya que las respuestas no especificadas se asignaron en su totalidad a las mujeres que no habían tenido hijos. En algunos grupos de edad este tipo de respuesta alcanza el $23 \%$, originando por tanto graves problemas (cuadro 4).

Tomando en cuenta lo anterior, se reducen las posibilidades de estimar de modo confiable la mortalidad regional y urbanorural. De hecho, tal parece que los datos más precisos corresponden a la mortalidad infantil. Incluso, las tablas de mortalidad urbano-rural de México construidas en el Bureau of Census de Estados Unidos (Rowe, 1982) para 1969-1971 parten de los niveles de mortalidad infantil observados en cada zona y del uso del patrón nacional para el resto de las edades, así como del sistema lógito de W. Brass ${ }^{6}$ (Brass, 1968).

De acuerdo con esta evaluación hemos decidido analizar únicamente algunas estimaciones de la mortalidad infantil urbanorural derivadas de las recientes encuestas. Antes, y como marco de referencia, se presenta un panorama general de la mortalidad en México.

\footnotetext{
${ }^{5}$ En Durango, las estadísticas vitales señalan en 1976 una mortalidad infantil de $31 \%$. Según el censo, se alcanzarla un valor de $87 \%$, lo que equivaldrfa a una omisión de las cifras del Registro Civil del $180 \%$. Esto es difícil de creer, a pesar de las deficiencias de las estadisticas vitales (Camposortega, 1986).

6 La aplicación de esta metodologfa es una alternativa muy interesante en vista de la poca confiabilidad de la información. Sin embargo, no permite estimar las características propias de la mortalidad por tamaño de localidad (sobremortalidad relativa en algunas edades, etc.).
} 
Gráfica 4

México: Mortalidad infantil urbana y rural, 1952-1976

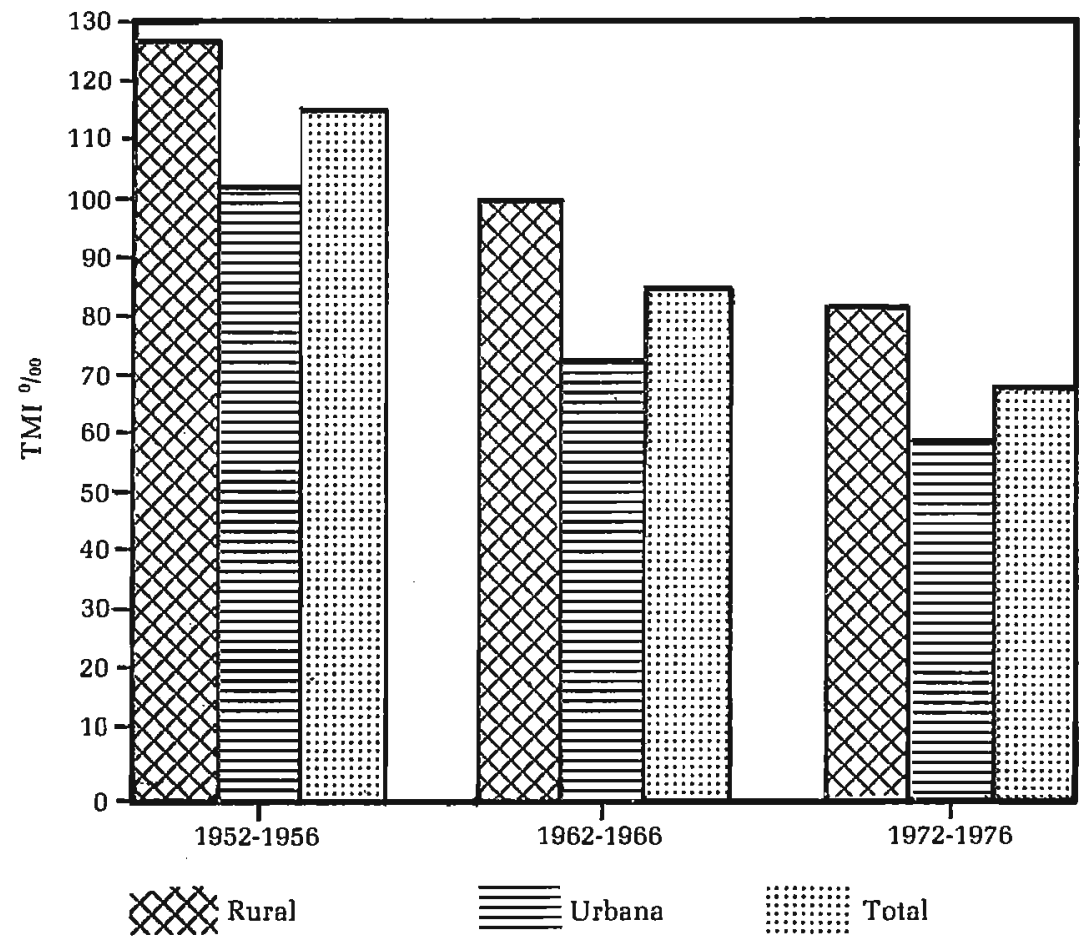

CUADRO 4

México: hijos nacidos vivos publicados y estimados con base en la distribución de los no especificados

\begin{tabular}{|c|c|c|c|c|}
\hline \multirow[b]{2}{*}{ Edad } & \multicolumn{2}{|c|}{ Hijos nacidos vivos } & \multicolumn{2}{|c|}{ Diferencias } \\
\hline & Estimados & Censo & Absolutas & $\%$ \\
\hline Total & 46286467 & 41804621 & 4481846 & 9.68 \\
\hline $15-19$ & 794668 & 766946 & 27722 & 3.49 \\
\hline $20-24$ & 4588892 & 3512164 & 1076728 & 23.46 \\
\hline $25-29$ & 6832294 & 5970532 & 861762 & 12.61 \\
\hline $30-34$ & 7955178 & 7266787 & 688391 & 8.65 \\
\hline $35-39$ & 9422815 & 8724545 & 698270 & 7.41 \\
\hline $40-44$ & 8777898 & 8173361 & 604537 & 6.89 \\
\hline $45-49$ & 7914722 & 7390286 & 524436 & 6.63 \\
\hline
\end{tabular}

-Fuente: Camposortega [1982]. 


\section{Evolución de la mortalidad en México en 1940-1980}

El rasgo característico de la evolución de la mortalidad en México de 1940 a 1980 es el considerable descenso experimentado, el cual se aprecia en cualquier indicador que se utilice. De esta forma, la tasa de mortalidad infantil pasa de $168 \%$ on 1940 , a $90 \%$ en 1960, y a $53 \%$ en 1980 y la esperanza de vida al nacimiento, de 40.4 años en 1940 , a 57.8 en 1960 , y a 66.2 en 1980.

Las mayores ganancias se presentan entre 1940 y 1960 , en especial en la década de los cincuenta, donde se ganan a la muerte cerca de 9.5 años, de tal forma que la esperanza de vida pasa de 48.4 años en 1950 a 57.9 en 1960 . De 1960 a 1970 los incrementos son mucho más lentos, con lo que en 1970 se llega a los 61 años. A partir de 1970, las ganancias retoman un nuevo impulso, por lo que en el decenio la esperanza aumenta poco más de 5 años, llegando a 66.3 años en 1980.

Sobre esta evolución conviene destacar tres aspectos. ${ }^{7}$ Uno, las ganancias sorprendentes en la esperanza de vida: durante 20 años se consiguen aumentos anuales cercanos a la unidad. En comparación, los paises europeos nunca tuvieron ganancias anuales mayores a 0.5 años (Stolnitz, 1955), y muy pocos países subdesarrollados han logrado incrementos similares en períodos de la misma extensión. ${ }^{8}$ Dos, que el descenso de la mortalidad no se explica exclusivamente por mejoras significativas en el nivel de vida de la población; la importación de tecnología médica desempeña también un papel muy importante. Y tres, que a pesar de las enormes reducciones, los niveles actuales de mortalidad son todavía muy altos comparativamente, de tal suerte que la mortalidad infantil de 1980 es siete vẹces mayor que la observada en Suecia - Japón, e incluso casi tres veces superior a la de países como Cuba o Costa Rica.

La comparación de la evolución observada en México con la de otras regiones permite apuntar que la esperanza de vida nacional ha presentado niveles superiores a los observados en los pafses subdesarrollados y en el promedio mundial, si bien las distancias respecto a las regiones desarrolladas son aún considerables: poco más de 10 años en 1960 y alrededor de 6 años en 1980.

De los dos sexos, el femenino es el que ha obtenịdo las mayo-

7 Para América Latina, A. Palloni apunta las dos primeras conclusiones (Naciones Unidas y Organización Mundial de la Salud, 1983).

8 Uno de los descensos más rápidos de mortalidad se presenta en Sri Lanka, donde la esperanza de vida al nacimiento se incrementa de 42.7 años en 1946 a 62.9 en 1963, logrando así ganancias anuales de 1.2 años en 17 años (Meegama. S.A., 1985). 
res ganancias; así, de 1940 a 1950 las mujeres incrementan su esperanza de vida 9 años, al pasar de 41.7 a 50.7 años, mientras que los hombres la aumentan 7.6 años, de 39.1 a 46.7 . Hacia 1960, las mujeres vivían en promedio 59.7 años y los hombres 56.0. En 1970 , las mujeres alcanzan los 63.1 años y los hombres 59.0 años, conservándose la mayor velocidad en el sexo femenino. Finalmente, de 1970 a 1980 el aumento es mucho menos intenso en los hombres que en las mujeres, de tal suerte que ellos incrementan su esperanza de vida en 4 años para llegar a 63.2 años y ellas más de 6, para alcanzar los 69.4 años en 1980.

Con relación a la estructura de la mortalidad de los países desarrollados occidentales, la mortalidad mexicana se caracteriza por niveles elevados en la población infantil, de 5 a 9 años, y en la adulta (20 a 40 años); niveles muy altos de mortalidad preescolar (1 a 4 años) y, por el contrario, niveles inferiores en la mortalidad de la tercera edad. La alta mortalidad de los niños obedece a la elevada proporción de fallecimientos por enfermedades infecciosas (Pérez Astorga, 1988). En el caso femenino sobresale la comparativamente alta mortalidad de las niñas entre 1 y 5 años y en el de los hombres, el incremento comparativo de la sobremortalidad de los adultos (15 a 45 años) que se observa en 1980.

A través del tiempo la estructura mexicana ha sufrido algunas transformaciones, al registrarse descensos de diferente intensidad en los distintos grupos de edad y en los dos sexos, lo que ha originado un incremento de la sobremortalidad masculina. De esta forma, de situarse entre un 10 y $25 \%$ hacia 1940 , la sobremortalidad entre 15 y 44 años rebasa en 1980 el $80 \%$ y. en algunos grupos (15 a 34 años) el 130\%. La explicación de este hecho se encuentra en gran medida en las muertes violentas, las cuales son más frecuentes en el sexo masculino.

\section{Mortalidad urbano-rural}

En los países desarrollados las diferencias en la mortalidad de las zonas urbanas y rurales son apenas perceptibles, con niveles ligeramente más bajos en el campo. Anteriormente, sin embargo, las diferencias eran mucho más importantes, sobre todo antes del siglo Xx (Naciones Unidas, 1978). De esta forma, hacia 1841 la esperanza de vida masculina en Inglaterra se calculaba en 40 años, mientras que en Londres se estimaba en 35 años y en Manchester y Liverpool en 24 y 25 años respectivamente (Naciones Unidas, 1978).

En los países subdesarrollados, a pesar de las dificultades 
para establecer diferenciales regionales y urbano-rurales, se ha podido determinar una sobremortalidad en el medio rural que en algunos casos es importante. De tal suerte, según estudios realizados en 12 países latinoamericanos, $\mathrm{H}$. Behm mostró que en el campo la probabilidad de morir antes de los 2 años era, en dos tercios de los países analizados, superior en un 30 a $60 \%$ a la correspondiente de las ciudades [cuadro 5] (Behm, 1979). No obstante, existen algunas excepciones a este patrón, como Haití, pues la mortalidad infantil en Puerto Príncipe es muy superior a la del resto del país y las regiones rurales (Joseph, 1989).

CUADRO 5

Probabilidad de morir entre 0 y 2 años según población urbana y rural en algunos países de América Latina, 1968-1970

\begin{tabular}{|c|c|c|c|c|c|}
\hline \multirow[b]{2}{*}{ País } & \multirow[b]{2}{*}{ Periodo } & \multicolumn{2}{|c|}{$q(0,2)(0 / 00)$} & \multirow{2}{*}{$\begin{array}{c}\text { Sobremortalidad } \\
\text { rural } 0 \% 00\end{array}$} & \multirow{2}{*}{$\begin{array}{l}\text { Población } \\
\text { rural } \%\end{array}$} \\
\hline & & Urbana & Rural & & \\
\hline Bolivia & $1971-1972$ & 166 & 224 & 34.90 & 62 \\
\hline Perú & 1966-1967 & 132 & 213 & 61.40 & 40 \\
\hline Nicaragua & $1966-1967$ & 143 & 152 & 6.30 & 65 \\
\hline Guatemala & $1968-1969$ & 119 & 161 & 35.30 & 64 \\
\hline El Salvador & $1966-1967$ & 139 & 148 & 6.50 & 60 \\
\hline Honduras & $1969-1970$ & 113 & 150 & 32.70 & 69 \\
\hline Ecuador & $1969-1970$ & 98 & 145 & 48.00 & 59 \\
\hline R. Dominicana & $1970-1971$ & 115 & 130 & 13.00 & 60 \\
\hline Chile & $1965-1966$ & 84 & 112 & 33.30 & 25 \\
\hline Colombia & $1968-1969$ & 75 & 109 & 45.30 & 36 \\
\hline Costa Rica & 1968-1969 & 60 & 92 & 53.30 & 59 \\
\hline Paraguay & $1967-1968$ & 69 & 77 & 11.60 & 63 \\
\hline
\end{tabular}

Fuente: Behm (1979).

La evolución de la mortalidad infantil en América Latina según la condición de residencia, puede resumirse en tres etapas [Guzmán, 1984]:

Primera. Alta mortalidad con diferencias variables según el país.

Segunda. Descenso mayor de la mortalidad infantil urbana, de modo que las diferencias se amplian, y

Tercera. Descenso ligeramente superior de la mortalidad rural, disminuyendo, por tanto, las diferencias.

La evolución descrita por las dos primeras etapas se ajusta, según muestran los datos de la Encuesta Mexicana de Fecundidad, al comportamiento nacional. De esta forma es posible observar un incremento de las diferenciales urbano-rurales con el paso del 
tiempo. Así, la mortalidad infantil rural de las generaciones 19521956 rebasa a la urbana en un $19 \%$, en tanto que en las correspondientes a 1962-1966 y 1972-1976 la diferencia llega al 28\% [García y Garma, 1983). En la primera generación, la mortalidad infantil rural puede aproximarse en $126.3 \%$ y la urbana en 102.4 por mil, contra 115.3 del total nacional. Hacia 1962-1966 los valores descienden a $99.9,72.1$ y $84.9 \%$ y en 1972-1976 la mortalidad infantil en el campo estimada es del orden de $81.4 \%$ la urbana de $58.4 \%$ y la nacional de $67.3 \%$ (gráfica 4). Los valores de la mortalidad urbana y rural no corresponden exactamente a los datos captados por la encuesta, pues se calcularon con base en las diferencias citadas por García y Garma (1983) y en las tasas nacionales.

Según los resultados de la encuesta de 1976 para la cohorte 1970-1975, la mortalidad infantil rural alcanzaba un valor de $78 \%$, en tanto que la urbana de $56 \%$ (SPP, 1979). De acuerdo con estos datos, la diferencia se conserva en 28 por ciento.

Los datos de la Encuesta Nacional de Prevalencia en el Uso de Métodos Anticonceptivos corroboran los resultados de la encuesta de 1976. De esta forma, hacia 1975 las localidades de menos de 2500 habitantes presentan una tasa de mortalidad infantil de $82 \%$; las de 2500 a 20000 , de $71 \%$; las mayores de 20000 , excluyendo a las tres zonas metropolitanas del país, de $57 \%$, y México, Guadalajara y Monterrey, de 53\%o (Mojarro, et al., 1982). La sobremortalidad respecto a estas tres zonas metropolitanas es, entonces, del $58 \%$ en el primer caso, del $37 \%$ en el segundo y del $10 \%$ en el tercero.

Comparativamente con los datos nacionales, es posible observar en 1952-1956 una sobremortalidad rural del orden del $10 \%$ y una submortalidad urbana de 11\%, en 1962-1966 aquélla se incrementa a $18 \%$ y ésta a $15 \% \mathrm{y}$, finalmente, en 1972-1976, el primer valor pasa a $21 \%$ y el segundo a $13 \%$. En 1975, según datos de la encuesta de 1979, la sobremortalidad de las localidades de menos de 2500 habitantes aumenta a 26\%; las de 2500 a 20000 presentan una de $9 \%$, las localidades urbanas de $12 \%$ y las tres metrópolis de $18 \%$. El cambio en estos porcentajes, sin embargo, se encuentra influido por el incremento de la población urbana en el total nacional, lo que origina un mayor peso de esta mortalidad en el total y, por tanto, un incremento artificial parcial de la sobremortalidad rural. ${ }^{9}$

Estos resultados confirman la relación inversa, encontrada en

${ }^{9}$ Los porcentajes se ven también influidos por las diferencias entre las encuestas de 1976 y 1979. 
otros países de América Latina, entre mortalidad y tamaño de localidad. De hecho, las grandes ciudades se caracterizan por contar con las tasas de mortalidad más bajas. En el caso de México, la comparación entre los datos del Distrito Federal y los del país confirman esta afirmación (cuadro 6). De este modo, las diferencias en la esperanza de vida se han mantenido en alrededor de 3-4 años entre 1950 y 1980 . En el caso de la mortalidad infantil, las diferencias porcentuales entre estas regiones se han incrementado considerablemente, al pasar, en el caso de los hombres, del $11 \%$ en 1950 al $37 \%$ en 1970 y al $45 \%$ en 1980 , y en el de las mujeres, del $-5 \%$ en 1950 , al $26 \%$ en 1970 y al $41 \%$ en 1980 . En 1950 la mortalidad infantil nacional alcanzaba un valor de $132 \%$ y la del Distrito Federal de $126 \%$, en tanto que en 1980 llegaba en el primer caso al $53 \%$ y a $30 \%$ on el segundo.

A pesar de los problemas de información, el análisis de los datos provenientes de las estadísticas vitales muestra algunos hechos interesantes [cuadro 2), como la considerable sobremortalidad rural en el Estado de México en 1980, donde la tasa rural es tres veces superior a la urbana: 12.5 contra $4.3 \%$. Este hecho muestra las desfavorables condiciones en que se encuentra el área rural de esta entidad (Camposortega y Mejía, 1987), aunque en parte también puede explicarse por el traslado de registros del estado al Distrito Federal.

Las diferencias urbano-rurales tienen su explicación en el papel que la agricultura desempeña en el sistema económico mexicano y cuyas principales funciones son proveer alimentos y fuerza

CUADRO 6

México y Distrito Federal: esperanzas de vida al nacimiento y tasas de mortalidad infantil, 1950-1980

\begin{tabular}{lrrrr}
\hline Regióniparámetro & 1950 & 1960 & 1970 & 1980 \\
\hline México & & & & \\
Esperanza de vida, hombres & 46.7 & 56.0 & 59.0 & 63.2 \\
Esperanza de vida, mujeres & 50.7 & 59.7 & 63.1 & 69.4 \\
Mortalidad infantil, hombres & 144.6 & 100.6 & 85.4 & 58.4 \\
Mortalidad infantil, mujeres & 118.7 & 79.4 & 67.8 & 47.5 \\
& & & & \\
Distrito Federal & & & & \\
Esperanza de vida, hombres & 49.1 & 60.0 & 62.2 & 67.8 \\
Esperanza de vida, mujeres & 53.2 & 63.3 & 65.5 & 72.3 \\
Mortalidad infantil, hombres & 128.6 & 73.4 & 53.7 & 32.1 \\
Mortalidad infentil, mujeres & 124.1 & 69.5 & 50.4 & 28.1 \\
\hline \multicolumn{1}{c}{ Fuente: Camposortega (1988b). } & & & &
\end{tabular}


de trabajo barata para el desarrollo de la sociedad. Estas características han originado una amplia diferenciación en las poblaciones y condiciones de vida urbano-rurales. De esta forma, y en términos generales, la población que habita en localidades urbanas se caracteriza por contar con mayores ingresos, tener mejores oportunidades de empleo, estar más escolarizada y, en fin, contar con mejores niveles de vida. Asimismo, y en vista de las condiciones estructurales diferenciadas, la población urbana se beneficia de mejores servicios médicos y sociales, como agua potable, drenaje, etcétera.

Estas condiciones, entonces, explican las diferencias en mortalidad entre ambas zonas y ponen de manifiesto los grandes esfuerzos que deben realizarse si se quieren disminuir los niveles de mortalidad rural.

\section{Consideraciones finales}

A pesar de la importancia que tienen los estudios sobre las diferencias de mortalidad urbano-rurales, en México este tema ha sido poco estudiado y prácticamente no existen trabajos específicos al respecto.

En parte, esta situación se explica por la escasa y deficiente información, la cual, en el caso de las estadísticas vitales, subregistra la mortalidad rural y, en el de las encuestas, sólo ha permitido calcular convenientemente la mortalidad en las primeras edades.

El análisis de los pocos datos disponibles, sin embargo, ha permitido distinguir las principales características de la mortalidad urbano-rural. Al respecto, cabe resaltar:

La relación inversa entre tamaño de localidad y mortalidad infantil. Comparativamente cón los niños de las tres grandes ciudades nacionales, los que habitan en localidades rurales de menos de 2500 habitantes sufren una sobremortalidad del 58\% en el primer año. La mortalidad infantil hacia 1975 en el primer caso era de $53 \%$, contra $82 \%$ en las zonas rurales.

En la actualidad, cada año nacen en el campo mexicano cerca de 800000 niños, de los cuales casi 50000 fallecen antes de su primer cumpleaños. Si las localidades rurales tuvieran los niveles de mortalidad de la capital, cada año podrían evitarse 20000 defunciones de niños menores de un año. De hecho, y con un desarrollo más equitativo, estas muertes no deberían ocurrir. Incluso, si los niveles descendieran a los del promedio nacional, las defunciones infantiles evitadas llegarían a 10000 cada año.

Los diferenciales urbano-rurales se han incrementado en los últimos decenios. De este modo, en los cincuenta la mortalidad in- 
fantil rural superaba a la urbana en menos de $20 \%$, en tanto que en los setenta la diferencia era del $28 \%$. La comparación de los niveles de la capital con el total del país muestra, asimismo, un incremento significativo: de $5 \%$ en 1950 a $43 \%$ en 1980 . En 1950 , el Distrito Federal contaba con una tasa de mortalidad infantil de $126 \%$, contra $132 \%$ del país y en 1980 los valores se reducen a $30 \%$ y $53 \%$, respectivamente.

El incremento de los diferenciales es una clara muestra del empobrecimiento relativo - respecto al total nacional- de los niveles de vida en el campo mexicano.

\section{Bibliografía}

Aguirre, A. y S. Camposortega [1981]. "Evaluación de la información básica sobre mortalidad infantil en México", Demografía y Economía, vol. XIV, núm. 4, El Colegio de México, México.

Arriaga, E. (1967). "Rural Urban Mortality in Developing Countries: An Index for Detecting Rural Underregistration", Demography, vol. 4, núm. 1.

Benítez, R. (1962). "La población rural y urbana de México", Revista Mexicana de Sociología, vol. xxiv, núm. 3.

Behm, H. [1979]. "Socioeconomic Determinants of Mortality in Latin American", en Proceedings of the Meeting on Socioeconomic Determinants and Consequences of Mortality, Naciones Unides, Organización Mundial de la Salud, El Colegio de México, Nueva York.

Brass. W. (1971). Seminario sobre métodos para medir variables demográficas (fecundidad y mortalidad], serie DS, núm. 9, San José, Costa Ríca.

Brass, W. et al. (1968). The Demography of Tropical Africa, Princeton University Prees, Princeton, New Jersey.

, y K. Hill (1973). "Estimating Adult Mortality from Orphanhood", en Proceedings of the International Population Conference Lieja 1973, Unión Internacional para el Estudio Científico de la Población, vol. 3, Lieja.

Camposortega, S. (1984). "Evaluación y corrección de la población mexjcana censada en 1970", Revista de Estadística y Geografía, vol. 3, núm. 10, México.

(1986). "Evaluación de los datos sobre mortalidad captados en el censo de 1980", en Taller Nacional del Censo General de Población y Vivienda 1980. Memoria, vol. 2, Instituto Nacional de Estadfstica, Geografía e Informática, San Juan del Río, Querétaro.

(1988a). "El nivel y la estructura de la mortalidad en México, 19401980", en La mortalidad en México: niveles, tendencias y determinantes, El Colegio de México, México

(1988b). "Estimación de la mortalidad en México", en Población de México del siglo XX, Instituto Mexicano del Seguro Social, México. 
[1988c). L'analyse démographique de la mortalité au Mexique, 1940-1980, tesis de doctorado, Université Catholique de Louvain, Louvain-La-Neuve, Bélgica.

(1989). "Nuevas relaciones para la estimación indirecta de la mortalidad en las primeras edades", en III Reunión Nacional de Investigación Demográfica en México, Sociedad Mexicana de Demografía, Universidad Nacional Autónoma de México, México (en prensa).

y M.A. Mejía (1987). La marginación en el estado de México: un aporte a la planeación del desarrollo, El Colegio Mexiquense, Zinacantepec, México.

CEED [Centro de Estudios Económicos y Demográficos) (1981). Dinámira de la Población de México, El Colegio de México, México.

Coale, A. y P. Demeny (1966). Regional Model Life Tables and Stable Populations, Princeton University Press, New Jersey.

CPNPF (Coordinación del Programa Nacional de Planificación Familiar) [1980a). Encuesta Nacional de Prevalencia en el Uso de Métodos Anticonceptivos con Módulo Fecundidad/Mortalidad. Estimaciones de Fecundidad y Mortalidad, nivel nacional, México.

(1980b). Encuesta Nacional de Prevalencia en el Uso de Métodos Anticonceptivos con Módulo Fecundidad/Mortalidad. Documento Metodológico, México.

Cordero, E. (1968), "La subestimación de la mortalidad infantil en México", Demografía y Economía, El Colegio de México, México.

DGPF (Dirección General de Planificación Familiar) (1988). Reporte de los resultados de la Encuesta Nacional sobre Fecundidad y Salud, Secretaría de Salud, México.

García y Garma, I. (1983). "Algunos factores asociados con ]a mortalidad infantil en México", Demografía y Economía, vol. xVII, núm. 3(55), El Colegio de México, México.

Guzmán, J.M. (1984). "Mortalidad infantil y diferenciación sociogeográfica en América Latina, 1960-1980", Seminarios, El Colegio de México, México.

Hill, K. (1977). "Estimating adult mortality levels from information on Widowhood", Population Studies, vol. xxxI, núm. 1.

JSPF (Jefatura de Servicios de Planificación Familiar) (1983a). Encuesta Rural de Planificación Familiar 1981. Documento Metodológico, Instituto Mexicano del Seguro Social, México.

(1983b). Encuesta Rural de Planificación Familiar 1981. Resultados Nacionales, Instituto Mexicano del Seguro Social, México.

Joseph, F.P. (1989). Diferencias sociogeográficas de la mortalidad infantil en Haití, tesis de maestro en demografía, El Colegio de México, México.

Lailson, H. et al. (1979), Estimación de los nacimientos ocurridos durante el periodo 1950-1975. Dirección General de Estadística, Secretaría de Progamación y Presupuesto, México.

Meegama, S.A. (1985). "'The Mortality Decline in the 'Fast Declining' Developing Countries", en International Population Conference Floren- 
ce 1985, International Union for the Scientific Study of Population, Lieja, Bélgica.

Mojarro, O., et al. (1982). "Mortalidad", en J. Martínez M. (ed.), La revolución demográfica en México, 1970-1980, Instituto Mexicano del Seguro Social, México.

Monterrubio, M.I., y S. Camposortega (1986). "Evaluación de la cobertura y de los datos sobre estructura por edad y sexo del censo de 1980", en Taller Nacional del Censo General de Población y Vivienda de 1980, Instituto Nacional de Estadística, Geografía e Informática, SPP, México.

Naciones Unidas (1978). Factores determinantes y consecuencias de las tendencias demográficas, vol. I, ST/SDA/SER.A/50, Nueva York.

y Organización Mundial de la Salud (1983). Niveaux et tendances de la mortalité depuis 1950, ST/ESA/SER.A/74, Nueva York.

Núñez L. y L. Moreno (1986). México: proyecciones de población urbana y rural, 1980-2010, Academia Mexicana de Investigación en Demografía Médica, México.

Ordorica, M. y V. Medina (1986). "Evaluación de la información censal sobre fecundidad", en Taller Nacional del Censo General de Población y Vivienda, 1980, Instituto Nacional de Estadística, Geografía e Informática, SPP, México.

y J. Potter (1980). "Evaluation of the Mexican Fertility Survey 1976-1977", en Scientific Reports, núm. 21, World Fertility Survey, International Statistical Institute, Londres.

Partida, V. [1982). "Problemas en la estimación de la mortalidad a nivel regional en México: caso de] Distrito Federal y el Estado de México en 1970", Investigación Demográfica en México - 1980, Consejo Nacional de Ciencia y Tecnología, México.

Pérez Astorga, J. (1988). "Mortalidad por causas en México 1950-1980", en La mortalidad en México: niveles, tendencias y determinantes, El Colegio de México, México.

Rowo, P. (1982). Detailed Statịstics on the Urban and Rural Population of Mexico: 1950 to 2010, U.S. Bureau of the Census, Washington.

SPP (Secretaría de Programación y Presupuesto et al.) (1979). Encuesta Mexicana de Fecundidad, 3 vols., México.

Stolnitz, G. (1955). "A Century of International Mortality Trends: I", Population Studies, vol., 9, núm. 1. 